\title{
Editorial: Colorectal Cancer Screening Education in Medically Vulnerable Populations: the Role of the Journal of Cancer Education
}

\author{
John T. Vetto ${ }^{1}$
}

Published online: 1 October 2015

(C) American Association for Cancer Education 2015

The most effective medical journals, regardless of their particular area of focus, have at least three traits in common: they are readable, timely, and relevant. A review of any issue of the Journal of Cancer Education (JCE) demonstrates all these traits. Recent articles by our Editor-in-Chief, Art Michalek and your humble Deputy Editor have focused on increasing readability via accurate writing and review $[1,2]$. The consistent quality of our articles and our rising impact factor speak to the efforts of our expert contributors and our hard working editorial staff.

For evidence of timeliness, one need only consider the June 2015 issue of the journal (volume 30, number 2). A glance at the table of contents reveals that almost a quarter of the original research papers - 5 of $21-$ were on the subject of colorectal cancer (CRC) screening education [3-7]. There are few more pressing needs in cancer education today than this area; both the American Cancer Society and the US Preventative Task Force have stated that CRC is primarily a preventable disease, and screening for CRC in asymptomatic individuals can result in detection of early-stage CRC. Removal of adenomatous polyps in particular before they become an invasive $\mathrm{CRC}$ has been shown to reduce CRC incidence and mortality [8].

In the USA, colonoscopy is the most commonly used screening test, and adenomatous polyps are the most common screening finding. The American Cancer Society recommends

John T. Vetto

vettoj@ohsu.edu

1 Department of Surgery, Division of Surgical Oncology, Oregon Health \& Science University, 3181 S.W. Sam Jackson Park Rd., Mail code L619, Portland, OR 97239, USA starting screening with colonoscopy at age 50 in asymptomatic, normal-risk individuals and repeating the screen every 10 years if negative [9]. However, patients who are found to have adenomas are at increased risk for developing "interval cancers"-metachronous adenomas or cancers - within those 10 years. Accordingly, for patients in whom screening colonoscopy resulted in polypectomy, evidence-based guidelines are used to determine the interval at which this higher risk population should be rescreened.

The most common guidelines for surveillance colonoscopy after screening and polypectomy are those of the US MultiSociety Task Force on Colorectal Cancer (USMTF). These guidelines are based on the number and pathology of the lesions found [10]. In order of increasing aggressiveness, adenomatous polyps can be classified as tubular, villous, and serrated. Serrated polyps are larger and tend to be right sided. It is now known that these lesions represent the most aggressive form of adenomas; $35 \%$ of colorectal cancer derives from serrated polyps. On a molecular level, serrated lesions are associated with a BRAF mutation that leads to hypermethylation of the MLH1 promoter and resultant silencing of the MLH1 DNA mismatch repair gene [11].

Because of the high risk of cancer for serrated polyps, the USMTF recommends an interval colonoscopy at 5 years if the lesion is less than $1 \mathrm{~cm}$ and not dysplastic, 3 years if it is larger than $1 \mathrm{~cm}$ or dysplastic, 1 year if multiple lesions are found, and in 3-6 months if the lesion was not completely resected [10].

The authors of the June JCE papers on CRC screening are to be particularly congratulated because their papers were relevant - they addressed the very real issues of CRC screening in underserved populations. Despite the availability of various CRC screening options, the fact is that half of all US adults aged 50 years or more are not up to date with national screening guidelines, and screening rates are particularly low 
among vulnerable groups, such as the medically underserved, racial/ethnic minorities, and recent immigrants [12].

One way to raise $\mathrm{CRC}$ screening rates in underserved populations may be to offer screening options that are more accessible and acceptable. Immune fecal occult blood testing (iFOBT) is a relatively new, cheaper, more accessible, yet still effective screening alternative, which is cost effective while still resulting in a $30 \%$ reduction in CRC mortality [12]. It may be both more acceptable than colonoscopy to immigrants and more practical for underserved patients, who are often treated in federally qualified health centers (FQHCs) where colonoscopy is often not available [13]. In one of the JCE papers, Gwede et al. reported on focus groups educating FQHC patients on the benefits of iFOBT for CRC screening. This timely study identified low knowledge and barriers and demonstrated the importance of teachable moments for both providers and patients in these health care settings [7].

Three of the five JCE papers focused on CRC screening education in Hispanic populations. Hispanics are an underserved population in this area of oncology; CRC is in the second most common cancer in Hispanic men and women, yet screening rates are low compared to non-Hispanic whites [14]. CRC incidence and death rates have fallen in the past 10 years across the USA, but not among Hispanics, especially Mexican-Americans, in whom CRC screening rates are particularly low, despite the fact that Hispanic men are at a higher risk of developing CRC than white men [15].

To tackle this difficult problem, all three of these JCE studies applied various aspects of tranformative learning theory, which can be central to education for underserved and minority populations. Transformative learning is the cognitive process of effecting change in a frame of reference. Frames of reference define our world, link learning to emotion, and may cause us to reject health behaviors that do not correspond to our particular values and ethnic experiences. In the transformative model, learning and subsequent behavioral changes occur when the educator and learner can shift to a frame of reference that is more inclusive and self reflective and integrates the experiences of others, especially those whom we understand and trust [16].

For example, Wilson et al. reported on a CRC male navigator program instituted at the University of Texas, San Antonio in 2011, targeted to Hispanic men. Over a period of 2 years, the program screened 370 of the 461 enrollees, resulting in an improved average life expectancy for the entire group of 6 months and cost savings of over $\$ 424,000$. In short, the authors used trusted navigators to effect transformative change and concluded that there were medical and financial benefits to navigator programs for CRC screening in underserved populations [4].

In the second of the JCE publications on CRC screening in Hispanics, Briant and colleagues focused on iFOBT kit knowledge and distribution at 47
Hispanic health fairs in rural Washington State. An inflatable walk-through colon displayed at the fairs generated a great deal of attention and interest. A remarkable $75 \%$ of the iFOBT kits the investigators distributed were returned for analysis, and logistic regression revealed that individuals most likely to follow through with screening were those with a regular health care provider. The authors concluded that given the opportunity for education and access, underserved populations can comply with CRC screening [3].

The third paper was a qualitative study of message targets for CRC screening in Latinos of Puerto Rican descent. Hennelly and colleagues explored the cultural paradox of machismo, which was a barrier to colonoscopy that prevented the discussion and mediation of screening-related fears in men: fears of procedure related pain, treatment-related burden following a cancer diagnosis, and suffering and death from disease. Using other elements of Hispanic male culture, particularly storytelling and optimism, the authors were able to develop a decision-making model for improving CRC screening rates in this population though transformative learning. The model is dependent on knowledge and - similar to the Briant findings - access [5].

Another feature of effective journals is they are not afraid to publish limited or even negative data, if the issue studied is important enough. CRC is now the second most common cancer in women, accounting for $9.4 \%$ of all cancer afflicting women worldwide. Iraqi women - both natives and US immigrants - have seen a steady rise in CRC incidence, reflecting worldwide dietary and cancer trends [8]. The last of the five July 2015 JCE papers on CRC screening was a pilot pre/post study by Jillson and colleagues of CRC screening knowledge in a cohort of Iraqi women living in suburban Washington DC. The authors reported that a single educational encounter had only modest success, with a resurgence of fear and anxiety at 1 month post the educational intervention. In the discussion section of the paper, the authors noted that their findings are likely generalizable to immigrant populations and underscore the importance of determining specific belief, attitude, and practice barriers for underserved populations. This is an example of the Platinum Rule in cancer education [17]; in designing cancer education interventions, we must seek first to understand what the target population is wanting and needing and the specific cultural framework in which success will occur. This often takes time, effort, and repeated attempts!

CRC screening saves lives, and the mandate for cancer educators is to develop and provide population-specific messaging and resources to increase CRC screening rates, especially among the medically vulnerable. As the July 2015 issue illustrates, the JCE is a powerful tool in this important endeavor. 


\section{References}

1. Vetto JT (2014) Short and sweet: a short course on concise medical writing. J Cancer Educ 29(1):194-195

2. Michalek AM (2014) Down the rabbit hole... advice to reviewers. J Cancer Educ 29(1):4-5

3. Briant KJ, Espinoza N, Galvan A, Carosso E, Marchello N, Linde S et al (2015) An innovative strategy to reach the underserved for colorectal cancer screening. J Cancer Educ 30(2):237-243

4. Wilson FA, Villarreal R, Stimpson JP, Pagan JA (2015) Costeffectiveness analysis of a colonoscopy screening navigator program designed for Hispanic men. J Cancer Educ 30(2):260-267

5. Hennelly MO, Sly JR, Villagra C, Jandorf L (2015) Narrative message targets within the decision-making process to undergo screening colonoscopy among Latinos: a qualitative study. J Cancer Educ 30(2):268-276

6. Jillson I, Faeq Z, Kabbara KW, Cousin C, Mumford W, Blancato J (2015) Knowledge and practice of colorectal screening in a suburban group of Iraqi women. J Cancer Educ 30(2):284-293

7. Gwede GK, Koskan AM, Quinn GP, Davis SN, Ealey J, Abdulla R et al (2015) Patients' perceptions of colorectal cancer screening tests and preparatory education in federally qualified health centers. J Cancer Educ 30(2):294-300

8. Edwards BK, Ward E, Kohler BA, Eheman C, Zauber AG, Anderson RN, Jemal A et al (2010) Annual report to the nation on the status of cancer 1975-2006, featuring colorectal cancer trends and impact of interventions (risk factors, screening, and treatment) to reduce future rates. Cancer 116(3):544-573
9. Smith RA, Manasssaram-Baptiste D, Brooks D et al (2014) Cancer screening in the United States. CA Cancer J Clin 64(7):30-51

10. Lieberman DA, Rex DK, Winawer SJ et al (2012) Guidelines for colonoscopy surveillance after screening and polypectomy: a consensus update by the US Multi-Society Task Force on Colorectal Cancer. Gastroenterology 143:844-857

11. Vu HT, Lopez R, Bennett A, Burke CA (2011) Individuals with sessile serrated polyps express an aggressive colorectal phenotype. Dis Colon Rectum 54(10):1216-1223

12. Quintero E, Castells A, Bujanda L, Cubiella J et al (2012) Colonoscopy versus fecal immunochemical testing in colorectalcancer screening. N Engl J Med 366(8):697-706

13. Khankari K, Eder M, Osborn CY, Makoul G, Clayman M, Skripkauskas S, Diamond-Shapiro L et al (2007) Improving colorectal cancer screening among the medically underserved: a pilot study within a federally qualified health center. J Gen Intern Med 22(10):1410-1414

14. American Cancer Society (2014) Cancer facts \& figures 2014. American Cancer Society, Atlanta. Available at http://www. cancer.org/acs/groups/content/2epidemiologysuveillnace/ documents. Accessed 2 Sept 2015

15. Center for Disease Control and Prevention (CDC) (2012) Cancer screening, United States-20120. MMWR Morb Mortal Wkly Rep 61(3):41-45

16. Mezirow J (1997) Transformative learning: theory to practice: new directions for adult and continuing education. Jossey-Bass, San Francisco

17. Vetto JT (2015) Reflections: cancer education and the "platinum rule". J Cancer Educ 